\title{
Psychose et Guérison. Le Chemin de la Compassion
}

Philippe Huguelet

Correspondance:

Dr Philippe Huguelet

Hôpitaux Universitaires de Genève

Département de Psychiatrie

Service de Psychiatrie Adulte

Rue du 31-Décembre 36

CH-1207 Genève

Tél. 0223823119

philippe.huguelet@hcuge.ch

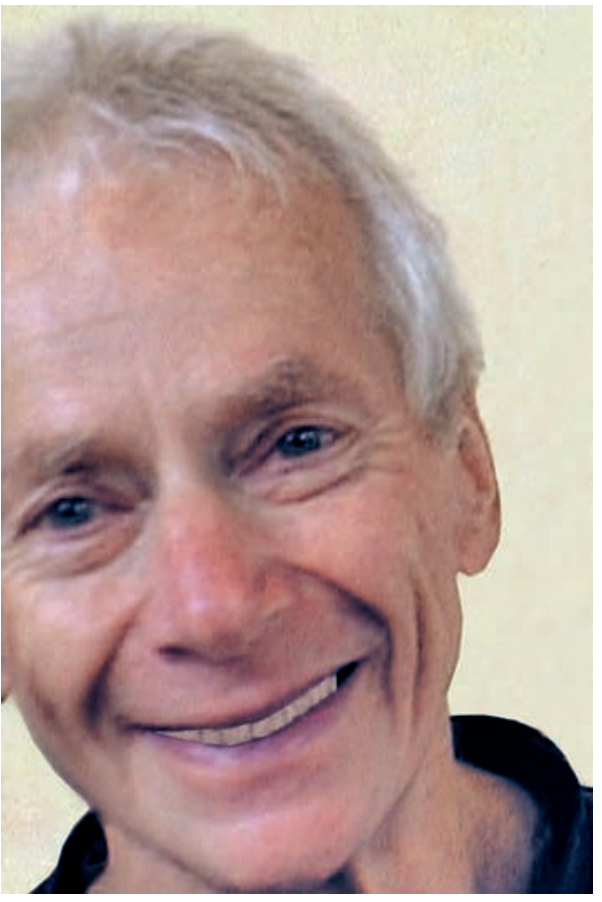

Edward M. Podvoll,

fondateur du projet «Windhorse».

Le soin aux patients souffrant de troubles psychotiques reste complexe. Les traitements pharmacologiques sont la plupart du temps indispensables, quoique largement insuffisants. Par conséquent, l'approche psychosociale qui l'accompagne représente un défi pour le clinicien. Dans ce sens, des travaux récents mettent en exergue le principe du rétablissement, qui implique de prendre le patient comme acteur de son projet de vie et ce même si d'éventuels symptômes persistent [2]. Malgré cela, de nombreux systèmes de soins s'obstinent à perpétrer une psychiatrie paternaliste, voire asilaire dans laquelle le patient n'a qu'un rôle passif.

\section{Le rétablissement}

C'est dans ce contexte que s'inscrit le livre d'Edward M. Podvoll. Ce médecin fut formé au traitement des psychoses par Harold Searles à Chesnut Lodge, aux Etats-Unis [3]. Fort de cet enseignement psychanalytique, il se tourna ensuite vers la méditation bouddhiste puis créa en
1981 le projet Windhorse dans le but de soigner des patients souffrant de psychose, en impliquant notamment des préceptes bouddhistes. Le présent ouvrage décrit cette démarche. Il consiste en la traduction d'un livre paru en 1990, complété dans cette édition par deux annexes.

\section{Phénoménologie}

Une première partie expose les situations de personnages historiques ayant décrit leurs propres épisodes psychotiques, spontanés ou consécutifs à la prise de substances. L'auteur nous amène à une compréhension phénoménologique intime de ces expériences. De manière très convaincante, il décrit le début de la psychose, caractérisée par la perte de contrôle. «On ne peut ni s'arrêter, ni commencer; une sorte de vaste système d'inertie a disparu [...]. C'est une zone de choc, l'expérience universelle, [...] l'état second» (p. 157). De cet «état second» naissent ce que l'auteur appelle les micro-opérations, p.ex. l'accélération des idées, leur répétition, multiplication, prolifération ... Dans cet état subsistent des «îlots de clarté», desquels la reprise de contact avec le monde va être envisagée dans le traitement. «Il n'est pas sot de dire que c'est l'hallucination qui rend fou et non pas la folie qui donne l'hallucination ...» (p. 152). Très justement, l'auteur observe que ce tableau clinique impose deux réponses possibles: celle de se déchaîner ou celle de devenir apathique.

C'est à cette phénoménologie que le thérapeute va être confronté dans son contact aux patients. «Le retour à la santé ne peut se produire qu'au sein d'un environnement sain, dans une atmosphère de compassion et de respect, qui peut favoriser le déroulement des étapes de la transformation» (p. 139). Podvoll mentionne une situation illustrative au XIX ${ }^{\mathrm{e}}$ siècle: «[...] pour atténuer cette influence médicale, [le patient] milita de plus en plus pour que les prêtres participent aux soins des aliénés» (p. 83).

\section{Le projet Windhorse}

La deuxième partie du livre décrit le développement, puis les principes de la prise en charge Windhorse. La synchronisation entre le corps 


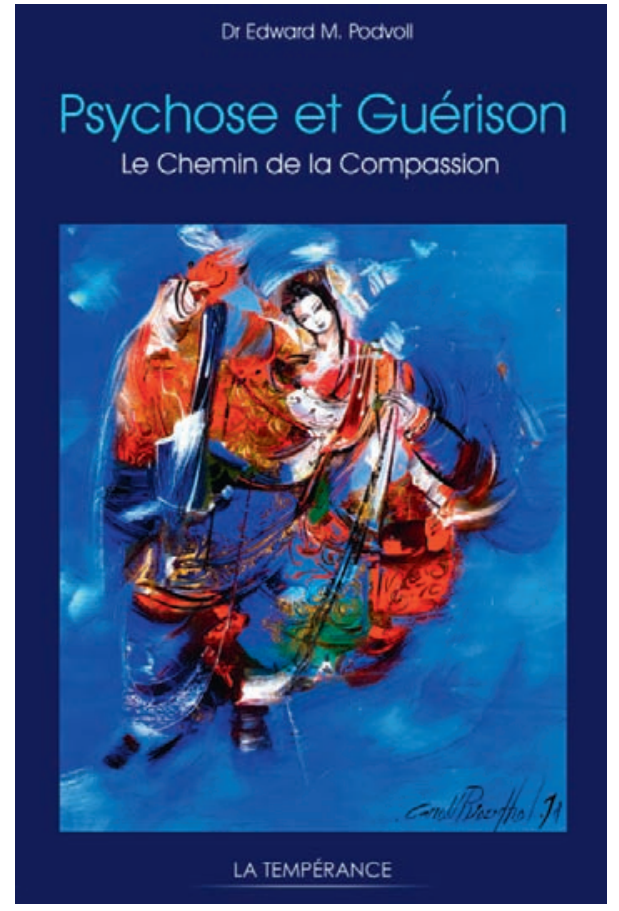

et l'esprit en est la notion clé. «En quelques mots, la synchronisation corps-esprit consiste à être conscient du corps, de l'esprit et, en même temps, de l'activité que l'on entreprend» (p. 249). L'auteur appelle cela la «méditation en action», laquelle peut consister en des exercices respiratoires. Podvoll introduit également le concept «d'assistance de base», accompagnement des patients organisé sous forme de plages de trois heures où le soignant accomplit diverses démarches. Cela dans le contexte d'une équipe où les intervenants se consacrent au rétablissement du patient, en relation directe avec les «îlots de clarté». L'action thérapeutique consiste principalement à «être présent», donc à être attentif et en même temps discret. Cette pratique s'inspire de la compassion enseignée dans la tradition thérapeutique bouddhiste (donner et recevoir). «En inspirant, on «prend sous forme d'obscurité, de solitude et de claustrophobie, la souffrance d'une ou de plusieurs personnes. Sur l'expiration, on envoie généreusement vers l'extérieur la notion de santé fondamentale, en l'accompagnant d'une sensation de fraîcheur, de légèreté et de présence amicale» (p. 277). Dans ce contexte thérapeutique, l'amitié peut apparaître entre le thérapeute et le patient, d'une manière peu en phase avec les techniques occidentales, mais tout en même temps semble-t-il très bien maîtrisée.

Ces démarches favorisent la responsabilité des patients, en les faisant avancer dans leur chemin vers le rétablissement. Destinée aux thérapeutes, mais aussi aux familles de patients, cette prise en charge s'appuie donc sur la tradition bouddhiste, notamment par la «dissolution de l'égocentrisme» et de sa territorialité, sa possessivité. «Enlever progressivement les œillères restrictives de l'ego apporte une meilleure perception du monde sensoriel et permet de reconnaître le sens du sacré et de la dignité dans la vie ordinaire. En cela, j'ai découvert le traitement le plus puissant pour la guérison de la psychose, ainsi qu'une inspiration majeure pour impulser chez quelqu'un un mouvement d'allégeance vers la santé» (p. 316).

\section{L'approche psychothérapeutique}

Dans la dernière partie de l'ouvrage, l'auteur décrit son analyse personnelle avec Searles, qui dura sept ans. Il nous donne des clés intéressantes sur la manière dont il a modifié la cure pratiquée par son Maître pour l'appliquer dans son projet, qui implique aussi un suivi psychothérapeutique. Il peut ainsi faire un lien entre l'expérience du transfert en jeu dans le traitement analytique et «l'interpénétration émotionnelle» décrite par les bouddhistes. S'ensuit enfin une discussion sur cette dialectique «identité vs. transcendance du sentiment de soi».

Pour un psychiatre habitué aux traitements «bio-psycho-sociaux» contemporains, maniant donc à la fois la psychopharmacologie et l'approche humaniste, la lecture d'un tel ouvrage ramène forcément à des sentiments mitigés. D'abord sur la forme: l'auteur adopte à la fois une écriture assez confuse, mais en même temps dirigée vers un but très précis. A ce sujet d'ailleurs, pourquoi commencer un tel livre par des témoignages historiques? Podvoll veut sans doute nous faire comprendre par là que l'approche d'un patient psychotique impose une capacité d'empathie importante, de même qu'une bonne compréhension des mécanismes en jeu.

\section{Les médicaments}

Il écrit par contre à quelques reprises des lignes parfois caricaturales, voire simplistes. Par exemple, la description phénoménologique mentionnée plus haut est suivie d'une théorisation un peu folklorique: «[...] lorsque le «cocktail» produisant la psychose engendre un déséquilibre neuro-électrique, cela réquisitionne et mobilise les rythmes corporels intrinsèques et inconscients. Ce déséquilibre amène les rythmes à la surface [...]» (p. 196). Aussi, sa position face aux traitements antipsychotiques est à la fois justifiée dans le contexte des années 70, mais tout en même temps peu «scientifique». Il écrit par exemple: «On sait actuellement que la thérapeutique médicamenteuse ne suffit pas à traiter 
la psychose et qu'elle n'a pas d'effet sur les phénomènes particuliers tels que les voix, les visions et les délires» (p. 237). Cette simple phrase combine un élément parfaitement exact et un autre complètement faux! Sa manière de décrire comment baisser le traitement est plutôt juste quoique sommaire au vu des connaissances récentes [4]. Cela n'empêche pas Podvoll de décrire quelque chose de profondément vrai, par ailleurs évoqué dans d'autres contextes [5]: la présence d'un environnement non stressant permet aux patients de diminuer le dosage de leurs médicaments.

Enfin, une limite du programme Windhorse est qu'il n'a jamais fait l'objet de recherches selon des critères scientifiques établis. Mais le but visé dans un tel programme est-il vraiment du ressort de la recherche? Peut-on pleinement mesurer le bonheur, la réalisation de soi? Il est des situations où on peut se rendre compte qu'une prise en charge fonctionne, sans étude randomisée ...

\section{En conclusion}

La pertinence de l'approche humaniste teintée de bouddhisme du projet Windhorse nous paraît établie. La dimension scientifique qui la soustend, de même que le regard porté sur les médicaments sont par contre encore largement discutables. Selon nos informations récentes, le projet Windhorse a survécu au décès en 2003 de son initiateur. Après quelques contacts avec les respon- sables actuels, il nous semble que le programme s'est maturé dans le sens d'une meilleure intégration entre la prise en charge et le traitement pharmacologique. Quoi qu'il en soit, le lecteur (qu'il soit médecin, thérapeute d'une autre filière ou proche de patient) y trouvera nombre de clés susceptibles de l'aider à faire face aux patients souffrant de troubles mentaux sévères, dans une perspective de rétablissement. Aussi et surtout, il nous donne des éléments précieux pour discuter avec les patients et leurs proches de ces traitements qu'ils pensent être alternatifs à ceux de la psychiatrie traditionnelle.

\section{Références}

1 Podvoll EM. Psychose et Guérison. Le Chemin de la Compassion. Paris: Edition de la tempérance; 2008.

2 Huguelet P. Le rétablissement, un concept organisateur des soins aux patients souffrant de troubles mentaux sévères. Schweiz Arch Neurol Psychiatr. 2007;158:271-8.

3 Searles H. L'effort pour rendre l'autre fou. Paris: Gallimard; 1977.

4 Calton T, Ferriter M, Huband N, Spandler H. A systematic review of the Soteria paradigm for the treatment of people diagnosed with schizophrenia. Schizophrenia Bull. 2007;34:181-92.

5 Hogarty GE. Expressed emotions and schizophrenic relapse: implications from the Pittsburg Study. In: Alpert M (ed.). Controversies in Schizophrenia. New York: Guilford Press; 1985. 